\title{
TGF-ß1-induced epithelial-mesenchymal transition increases fatty acid oxidation and OXPHOS activity via the p-AMPK pathway in breast cancer cells
}

\author{
QIAN-QIAN LIU ${ }^{1,2^{*}}$, HONG-YU HUO ${ }^{1,3^{*}}$, SONG AO $^{1,2^{*}}$, TING LIU $^{1,2}$, LI YANG $^{1,2}$, ZAI-YI FEI $^{1,2}$, \\ ZHI-QIANG ZHANG ${ }^{3}$, LEI DING ${ }^{1,2}$, QING-HUA CUI ${ }^{1,2}$, JIE LIN ${ }^{1,2}$, MIN YU ${ }^{1,2}$ and WEI XIONG ${ }^{4,5}$ \\ ${ }^{1}$ Department of Biochemistry and Genetics, School of Life Sciences, Yunnan University; \\ ${ }^{2}$ Key Laboratory for Biochemistry and Molecular Biology of High Education in Yunnan Province, \\ Yunnan University, Kunming, Yunnan 650091; ${ }^{3}$ Chifeng Blood Centre, Chifeng, Inner Mongolia, Chifeng 024000; \\ ${ }^{4}$ Department of Biochemistry and Molecular Biology, College of Basic Medical Sciences, Dali University; ${ }^{5}$ Key Laboratory for \\ Clinical Biochemistry of High Education in Yunnan Province, Dali University, Dali, Yunnan 671000, P.R. China
}

Received January 16, 2020; Accepted May 26, 2020

DOI: $10.3892 /$ or.2020.7661

\begin{abstract}
Breast cancer is the most common malignancy in women, and metastasis is the leading cause of death in breast cancer patients. Previous studies have shown that epithelial-mesenchymal transition (EMT) is involved in the metastasis of breast cancer, but the metabolic reprogramming and regulation mechanisms involved in the EMT process are still unclear. In the present study, we successfully constructed an EMT cell model induced by transforming growth factor $\beta 1$ (TGF- $\beta 1$ ) treatment of MCF-7 cells at different times. The results showed that cell adhesion decreased, cell invasion increased and ATP levels increased in EMT MCF-7 cells treated with TGF- $\beta 1$. Furthermore, the expression of fatty acid synthase (FASN) was decreased, and the expression of key fatty acid $\beta$-oxidation enzymes (CPT1 and CD36) was elevated in treated cells compared to control cells. These results showed that the fatty acid oxidation pathway was enhanced. In addition, the expression of NADH:ubiquinone oxidoreductase subunit B8 (NDUFB8), mitochondrial transcription factor A (TFAM) and cytochrome c oxidase
\end{abstract}

Correspondence to: Professor Min Yu, Department of Biochemistry and Genetics, School of Life Sciences, Yunnan University, 2 Cuihu North Road, Kunming, Yunnan 650091, P.R. China

E-mail: yumin@ynu.edu.cn

Professor Wei Xiong, Department of Biochemistry and Molecular Biology, College of Basic Medical Sciences, Dali University, 02 Hongshen Road, Dali, Yunnan 671000, P.R. China

E-mail: xiongwei@dali.edu.cn

${ }^{*}$ Contributed equally

Key words: TGF- $\beta 1$, EMT, fatty acid oxidation, OXPHOS, p-AMPK, breast cancer subunit I (COXI) increased, and the mitochondrial DNA copy number and ROS levels were also significantly increased during TGF- $\beta 1$-induced EMT. These results indicated that mitochondrial oxidative phosphorylation (OXPHOS) activity was enhanced during EMT. In addition, we observed that the expression of p-AMPK was increased and ACC (Acetyl-CoA Carboxylase) was decreased during TGF- $\beta 1$-induced EMT in MCF-7 cells. Immunohistochemical analysis of clinical samples revealed high expression of FASN in epithelial cells that had high expression of E-cadherin, while high expression of CPT-1 was observed in mesenchymal cells that had high expression of vimentin. Results of the current study showed a metabolic transition in TGF- $\beta 1$-induced EMT in MCF-7 cells. This transition may regulate fatty acid oxidation and OXPHOS activity in EMT MCF-7 cells through the p-AMPK pathway. These data suggest that a metabolic transition that suppresses lipogenesis and favors energy production is an essential component of TGF- $\beta 1$-induced EMT and metastasis in breast cancer. This study thus provides a new strategy for identifying new therapeutic targets for breast cancer.

\section{Introduction}

Breast cancer is the most common malignant tumor affecting women's health worldwide. Due to the metastasis of breast cancer cells and sensitivity to chemotherapy, the incidence of breast cancer with approximately 1,7000,000 new cases each year remains alarmingly high, and the prognosis after surgery is poor (1). Most tumors are difficult to cure due to distant metastases, and some patients experience tumor recurrence after treatment. More than $90 \%$ of breast cancer-related deaths are associated with tumor cell metastasis, and metastatic sites usually include the bone, brain, liver and lung (2). Previous findings have shown that 54 genes, including MMP1, CXCL1 and PTGS2, are markers and mediate the metastasis of breast cancer cells to the lungs (3). Cancer stem cell-like cells (CSCs) promote tumor metastasis. Recent findings have shown that increasing the CD44v/CD44s ratio in breast cancer cells by 
regulating the expression of epithelial splicing regulatory protein 1 (ESRP1) leads to the promotion of lung metastasis without influencing cancer cell stemness (4). In addition, it has been reported that epithelial-mesenchymal transition (EMT) is associated with breast cancer metastasis (5).

EMT is a cellular process in which cells lose their epithelial characteristics and acquire mesenchymal features, which enable the cells to migrate more efficiently and invade the underlying mesenchyme (6). In cancer, EMT is associated with tumorigenesis, invasion, metastasis and resistance to chemotherapy (7). EMT can alter the expression of adhesion factors, enhance the migration and invasion of tumor cells, and ultimately transfer tumors away from the primary site to adjacent or distal organs. The growth and metastasis of primary tumors is controlled by the regulation of TGF- $\beta 1$, which induces EMT-related changes in cells (8). TGF- $\beta 1$ promotes tumorigenesis in the tumor microenvironment. Clinical data have shown that TGF- $\beta 1$ protects NIH3T3 fibroblasts (CAFs) from starvation-induced growth inhibition, mitochondrial damage and cell apoptosis (9).

EMT can induce cancer metastasis by promoting tumor malignancy, reprogramming cancer metabolism, and disrupting the extracellular matrix (10). Accumulating evidence has demonstrated that aberrant cancer metabolism can induce EMT through multiple pathological pathways (11). Most studies have focused on the relationship between glucose metabolism and EMT. Changes in lipid metabolism during EMT have also been reported (10).

The aim of the present study was to explore changes in fatty acid metabolism and regulatory mechanisms of TGF- $\beta 1$-induced EMT in breast cancer cells, which is of great significance for the clinical treatment of breast cancer.

\section{Materials and methods}

Reagents. Recombinant human TGF- $\beta 1$ was purchased from $\mathrm{R} \& D$ Systems. For in vitro cell studies, TGF- $\beta 1$ was reconstituted at $20 \mu \mathrm{g} / \mathrm{ml}$ in sterile $4 \mathrm{mM} \mathrm{HCl}$ containing $1 \mathrm{mg} / \mathrm{ml}$ human or bovine serum albumin and stored at $-20^{\circ} \mathrm{C}$. Then, the solution was further diluted in fresh medium for cell experiments. Primary antibodies against NADH-ubiquinone oxidoreductase chain 1 (ND1, 1:1,000; Anbo Biotechnology, Inc.), mitochondrial transcription factor A (TFAM, 1:500; Cell Signaling Technology, Inc.), pyruvate dehydrogenase (PDH, 1:500; CST), carnitine palmitoyl transferase 1A (CPT1A; 1:500; CST), fatty acid synthase (FASN; 1:500; CST), E-cadherin (1:500; CST), vimentin (1:500; CST), Snail (1:500; CST), HIF-1 $\alpha$ (1:1,000), NADH:ubiquinone oxidoreductase subunit B8 (NDUFB8, 1:1,000; Abcam), lactate dehydrogenase (LDH, 1:1,000; Abcam), and GAPDH (1:1,000; Abcam) were used in this study.

Patients and tissue samples. Breast cancer samples and matching normal tissues were obtained from 20 patients with breast cancer (19 females and 1 male; age range, 30.0-68.0 years) at the Thyroid and Breast Surgery of the First People's Hospital of Dali Autonomous Prefecture (Yunnan, China), between September, 2017 and December, 2018. The fresh tumor tissue specimens were excised from the tumors, and their corresponding adjacent normal tissues were taken $\geq 3 \mathrm{~cm}$ from the tumor edge. All specimens were stabilized by snap-freezing immediately in cryovials, immersed in liquid nitrogen and stored at $-80^{\circ} \mathrm{C}$ until analysis. All the patients were diagnosed for the first time and did not have a treatment history of chemotherapy or radiotherapy prior to surgery.

The study was approved by the Medical Ethics Committee of Dali University (no. 2016-10), and all patients signed an informed consent form.

Cell lines and cell culture. Human breast cancer MCF-7 cells were obtained from the Kunming Cell Bank of the Chinese Academy of Science (Kunming, China). The cell lines were cultured in high glucose DMEM (Gibco, Thermo Fisher Scientific, Inc.) containing 10\% fetal bovine serum (Biological Industries) and $1 \%$ penicillin-streptomycin (Gibco) at $37^{\circ} \mathrm{C}$ in a humidified incubator with $5 \% \mathrm{CO}_{2}$. The experiments were performed with cells in the logarithmic phase, and the cells were MAP-tested and free of mycoplasma contamination. To investigate the effect of metabolic reprogramming during TGF- $\beta 1$-induced EMT, MCF-7 cells were treated with $2 \mathrm{ng} / \mathrm{ml}$ TGF- $\beta 1$ at different times $(0,3,6$ and 9 days) and then further cultured withdrawal TGF- $\beta 1$ for 3, 6 and 9 days. After different treatments, the cells were observed and photographed for morphological alterations under an inverted light microscope.

RNA extraction and reverse transcription-quantitative polymerase chain reaction $(R T-q P C R)$. Total RNA was extracted from treated MCF-7 cells at different times using TRIzol $^{\circledR}$ reagent (Solarbio) according to the manufacturer's protocols and then quantified. The RNA $(2 \mu \mathrm{g})$ was used to generate first-strand DNA using M-MLV reverse transcriptase (Promega) at $42^{\circ} \mathrm{C}$ for $15 \mathrm{~min}$. For standard PCR, products were visualized using $1 \%$ agarose gels. All reactions were performed in triplicate on an ABI Prism 7300 Sequence Detection System (Applied Biosystems, Thermo Fisher Scientific, Inc.) using SYBR-Green (Takara Bio, Inc.) fluorescent dye, and the data were normalized to $\beta$-actin mRNA levels. PCR was performed under the following conditions: $94^{\circ} \mathrm{C}$ for $2 \mathrm{~min}$, followed by 40 cycles of $94^{\circ} \mathrm{C}$ for $30 \mathrm{sec}$, and $58^{\circ} \mathrm{C}$ for $40 \mathrm{sec}$. The primer sequences used are listed in Table I.

Determination of mtDNA content. Total genomic DNA was isolated from $2 \times 10^{6}$ TGF- $\beta 1$-treated MCF- 7 cells and at different times resuspended in 200- $\mu$ 1 PBS using a Tissue DNA kit (Omega) according to the manufacturer's protocol. mtDNA content was detected by RT-qPCR using ABI Prism 7300 Sequence Detection Systems according to the manufacturer's protocol (Applied Biosystems). RT-qPCR was conducted following the standard protocol using SYBR Premix Ex Taq (Takara): 1 cycle at $95^{\circ} \mathrm{C}$ for 2 min, 40 cycles at $95^{\circ} \mathrm{C}$ for $30 \mathrm{sec}$, and $58^{\circ} \mathrm{C}$ for $40 \mathrm{sec}$, followed by melt curve analysis. The D-loop content was normalized by $18 \mathrm{~S}$ rDNA. Specific primers were used to amplify the fragment of the mitochondrial D-loop region: 5'-GGGGAAGCAGATTTG GGTAC-3' (forward) and 5'-AGGGTGGGTAGGTTTGTT GG-3' (reverse), and the fragment of the nuclear genomic $18 \mathrm{~S}$ rDNA gene region: 5'-CAGGAAGGAAGGCTGGAAG-3' (forward) and 5'-CGGGAAATCGTGCGTGAC-3' (reverse).

Immunohistochemical (IHC) staining. Immunohistochemistry was performed using a horseradish peroxidase (HRP) detection 
Table I. Primer sequences used in RT-qPCR

\begin{tabular}{lcc}
\hline Target gene & Primer & Nucleotide sequence \\
\hline E-cadherin & $\mathrm{F}$ & 5'-GAACGCATTGCCACATACACT-3' \\
& $\mathrm{R}$ & 5'-TTCCATGACAGACCCCTAAA-3' \\
N-cadherin & $\mathrm{F}$ & 5'-TGGAGACATTGGGGACTTC-3' \\
& $\mathrm{R}$ & 5'-TGCTCACCACCACTACTTG-3' \\
Snaill & $\mathrm{F}$ & 5'-ATCCTCAACCCCACCGCCT-3' \\
& $\mathrm{R}$ & 5'-GCCTTTCCCACTGTCCTCA-3' \\
FASN & $\mathrm{F}$ & 5'-TGGGAGGAGTGTAAACAG-3' \\
& $\mathrm{R}$ & 5'-GAAGTAGGAGTGGAAGGC-3' \\
CPT1 & $\mathrm{F}$ & 5'-TCTTGGGGTGATGGTGTG-3' \\
& $\mathrm{R}$ & 5'-GTCAATAGTGAGGGTTTT-3' \\
ACC & $\mathrm{F}$ & 5'-TGTGCTGGTCTACATTCCTCC-3' \\
& $\mathrm{R}$ & 5'-TGATTTCTACCGTCCCTTCTG-3' \\
CD36 & $\mathrm{F}$ & 5'-CCGTGACATCAAGGAGAAGC-3' \\
& $\mathrm{R}$ & 5'-TACCGCAAGATTCCATACCC-3' \\
$\beta$-actin & $\mathrm{F}$ & 5'-CATGTACGTTGCTATCCAGGC-3' \\
& $\mathrm{R}$ & 5'-CTCCTTAATGTCACGCACGAT-3' \\
& &
\end{tabular}

system (Beyotime Institute of Biotechnology). The breast cancer and non-cancerous breast tissue samples were immediately fixed in $10 \%$ neutral-buffered formalin at room temperature for 24-48 h, and subsequently embedded in paraffin. The paraffin-embedded tissues were sectioned continuously at $4 \mu \mathrm{m}$. The slides were heated at $60^{\circ} \mathrm{C}$ for deparaffination for $1 \mathrm{~h}$, and endogenous peroxidase was blocked by immersion in hydrogen peroxide for $10 \mathrm{~min}$, followed by microwave pretreatment for antigen retrieval. Then, the slides were washed in PBS and incubated with E-cadherin and vimentin antibodies (1:1,000; Cell Signaling Technology, Inc.) at $37^{\circ} \mathrm{C}$ for $1 \mathrm{~h}$. The tissue sections were washed and incubated for $15 \mathrm{~min}$ at room temperature with preabsorbed biotinylated secondary antibodies specific for the types of primary antibodies used. The staining was visualized using 3,3'-diaminobenzidine (DAB) substrate-chromogen (Zymed Laboratories), and the slides were counterstained with hematoxylin. At high microscopy magnification (x400), five representative fields of view were selected, and the proportion of positively staining cells was counted in each visual field. The staining intensity scores were assigned as follows: 0 (negative), 1 (weak), 2 (moderate) and 3 (strong). The scores for the percentage of positive tumor cells were determined as follows: 1 (0-25\%), 2 (26-50\%), $3(51-75 \%)$ and 4 (76-100\%). The immunoreactive score (IRS) of each section was calculated by the product of the staining intensity and the percentage of tumor cells. According to the IRS, the staining patterns were divided into three classes: weak (IRS: 0-2), moderate (IRS: 2-4), and strong (IRS: 4-6).

Adenosine triphosphate measurements. A total of $3 \times 10^{5}$ cells were collected and resuspended in PBS. Then, $120 \mu \mathrm{l}$ of lysis buffer from ATP detection kit was added to the cells and incubated on ice for $30 \mathrm{~min}$ at $4^{\circ} \mathrm{C}$. The cell lysate was centrifuged at $1,000 \mathrm{x}$ g for $10 \mathrm{~min}$ at $4^{\circ} \mathrm{C}$, after which ATP levels in the supernatant were measured using an ATP detection kit (Beyotime). Bioluminescence was assessed on a LUMAT
LB9507 (EG\&G Berthold Technologies Inc.) and an ATP assay kit (Beyotime) was used to measure ATP levels in the supernatant. The relative light units (RLUs) were measured with a Pi-102 ATP fluorometer (Hygiena), and a standard curve was calculated to determine the ATP concentration (nmol/l). ATP content was normalized by using a BCA protein assay kit (Dingguo).

Matrigel invasion assay. Cell invasion was evaluated in Transwell chambers coated with $50 \mu 1$ of Matrigel (BD Biocoat) in serum-free DMEM at a dilution of 1:4. The upper and lower chambers were separated by polycarbonate membranes with $8-\mu \mathrm{m}$ pores (24-well inserts; Corning). MCF-7 cells were pretreated with TGF- $\beta 1(2 \mathrm{ng} / \mathrm{ml})$ for $24 \mathrm{~h}$. Then, the cells were collected and resuspended in serum-free DMEM and $5 \times 10^{4}$ cells were seeded in the upper chamber. The lower chambers contained $600 \mu \mathrm{l}$ of DMEM with $10 \%$ FBS as a chemoattractant. After incubating the cells for $24 \mathrm{~h}$ in a $37^{\circ} \mathrm{C}$ incubator, cells on the upper surface were removed with a cotton swab, and the invaded cells were fixed and stained with $0.5 \%$ crystal violet for $15 \mathrm{~min}$ and counted in five random fields at $\mathrm{x} 400$ magnification.

Western blot analysis. Cells were washed with ice-cold PBS and lysed with cell lysis buffer (Beyotime) containing protease inhibitors (Beyotime), and protein concentrations were quantified with an enhanced BCA protein assay kit (Dingguo). The total protein concentration of the extracted sample was adjusted to $2 \mu \mathrm{g} / \mathrm{ml}$. Total protein $(20 \mu \mathrm{g})$ were separated by $12 \%$ SDS-PAGE, and immunoblotting was performed on polyvinylidene fluoride (PVDF) membranes (Millipore). The membrane was blocked with 5\% fat-free milk in PBS for $2 \mathrm{~h}$ at room temperature and then incubated with primary antibodies at $4^{\circ} \mathrm{C}$ overnight. After 3 washes with Tris-buffered saline with Tween-20 (TBST), the PVDF membranes were incubated with secondary antibodies conjugated with HRP (1:1,000, Beyotime) for $2 \mathrm{~h}$ at room temperature. The antigen-antibody complexes were visualized with Immobilon Western Chemiluminescent HRP Substrate (Millipore). Then, the blots were imaged with a Tanon 5200 chemiluminescence imaging system.

Statistical analysis. Results are presented as the mean \pm standard error (means \pm SE) obtained from at least three independent experiments. Statistical significance was determined by the independent paired t-test or one-way analysis of variance with Dunnett's post hoc analysis using SPSS software (version 19.0; IBM Corp.). Pearson's correlation analysis was used to analyze the association of protein expression in breast cancer tissues. In all the statistical analyses, $\mathrm{P}<0.05$ was considered to indicate a statistically significant difference and all P-values were two-sided.

\section{Results}

TGF- $\beta 1$ induces EMT in MCF-7 cells. Findings have shown that TGF- $\beta 1$ induces EMT in lung cancer cells (12). To investigate the changes in energy metabolism during EMT in breast cancer cells, we constructed a TGF- $\beta 1$-induced EMT MCF-7 cell model. RT-qPCR was initially used to 
A

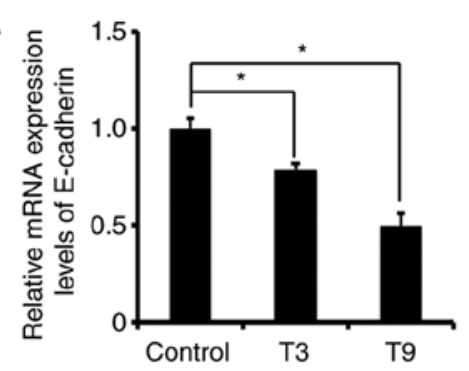

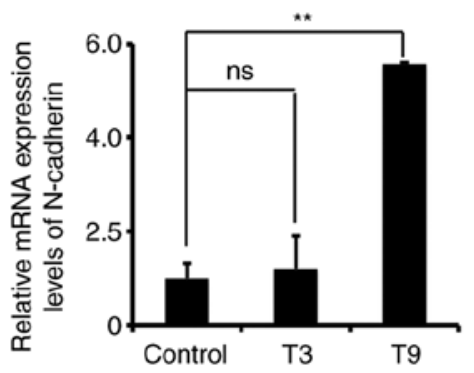

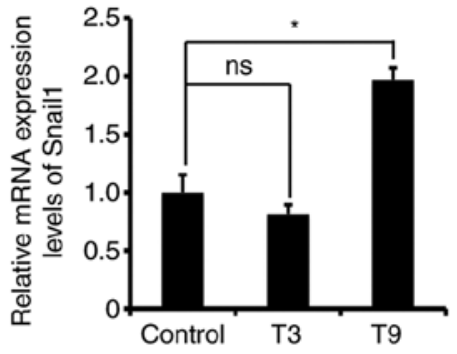

B

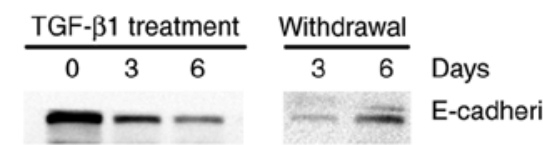

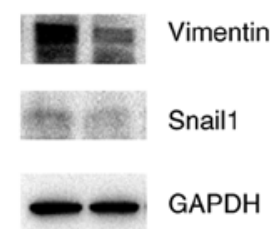

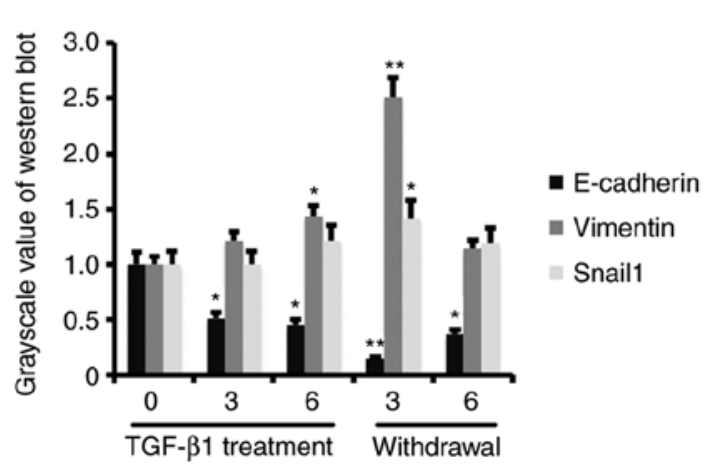

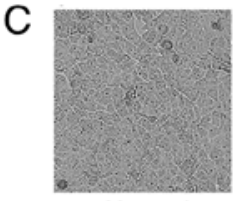

Normal

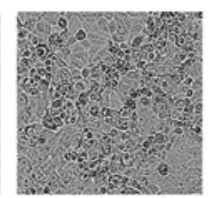

T3

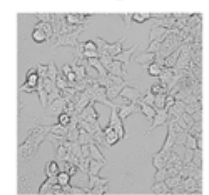

H3

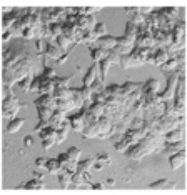

T6

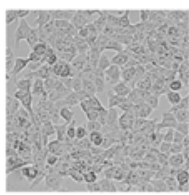

H6

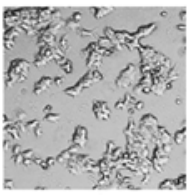

T9

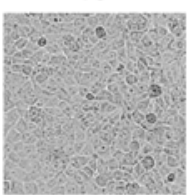

H9

Figure 1. TGF- $\beta 1$ induces EMT in MCF-7 cells. (A) mRNA expression of the EMT marker genes E-cadherin, N-cadherin and Snaill in MCF-7 cells treated with $2 \mathrm{ng} / \mathrm{ml}$ TGF- $\beta 1$ for 3 and 9 days. mRNA levels were normalized to the $\beta$-actin levels. (B) Protein expression of EMT marker genes in MCF-7 cells treated with $2 \mathrm{ng} / \mathrm{ml}$ TGF- $\beta 1$ for 0,3 and 6 days and then cultured withdrawal TGF- $\beta 1$ for 3, 6 and 9 days. (C) Cell morphology changes in MCF-7 cells during EMT and MET. $N$, normal; $T$, treated; $H$, recovered. The data are presented as the mean $\pm \mathrm{SE}(\mathrm{n}=3)$. ${ }^{*} \mathrm{P}<0.05,{ }^{* *} \mathrm{P}<0.01$ compared with the control cells.

detect the mRNA expression levels of EMT marker genes in MCF-7 cells after 3 and 9 days of treatment with $2 \mathrm{ng} / \mathrm{ml}$ TGF- $\beta 1$. The results showed that the mRNA expression level of E-cadherin decreased, and the expression of $\mathrm{N}$-cadherin and Snaill increased (Fig. 1A). We also examined the protein expression levels of EMT marker genes in MCF-7 cells treated with TGF- $\beta 1$ for 0,3 , and 6 days and further cultured them withdrawal TGF- $\beta 1$ for 3 and 6 days. The results showed that the protein expression level of E-cadherin decreased and $\mathrm{N}$-cadherin and Snaill increased after TGF- $\beta 1$ treatment. The opposite results were obtained after removal of TGF- $\beta 1$ (Fig. 1B). These results indicated that TGF- $\beta 1$ regulates the expression of EMT marker genes to promote the transformation of MCF-7 cells from epithelial to mesenchymal cells. In addition, we observed changes in cell morphology during the induction of EMT by TGF- $\beta 1$. As the induction time increased, the cells changed from a round shape to a long strip shape, and the cell adherence ability was reduced. After switching to normal medium, the original adherence ability of the cells was restored (Fig. 1C). These results confirmed that low concentrations of TGF- $\beta 1$ induced MCF-7 cells to transform from epithelial to mesenchymal cells.
Decreased cell proliferation, increased cell invasion and ATP levels during TGF- $\beta 1$-induced EMT. Previous findings have shown that TGF- $\beta 1$ affects cell proliferation, differentiation and EMT (13). We examined changes in cell proliferation, invasion and intracellular ATP levels in TGF- $\beta 1$-treated MCF-7 cells. We used MTS to detect the proliferative capacity of the cells, and the results showed that the addition of TGF- $\beta 1$ to MCF-7 cells significantly reduced the growth rate of the cells (Fig. 2A). Through cell invasion experiments, we found that TGF- $\beta 1$-treated MCF-7 cells were more invasive than control cells (Fig. 2B). We also measured intracellular ATP levels in MCF-7 cells at different times after TGF- $\beta 1$-induced EMT and TGF- $\beta 1$ removal. We found that there was no significant increase in the intracellular ATP content after TGF- $\beta 1$ treatment for 3 or 6 days. ATP levels increased significantly on the 9th day of TGF- $\beta 1$ treatment, while the ATP content decreased after the removal of TGF- $\beta 1$, and the intracellular ATP content did not change significantly with increasing culture time in the control cells (Fig. 2C).

Increased fatty acid oxidation and decreased fatty acid synthesis in TGF- $\beta 1$-induced EMT MCF-7 cells. Previous data showed that MCF-7 cells have increased ATP content 
A

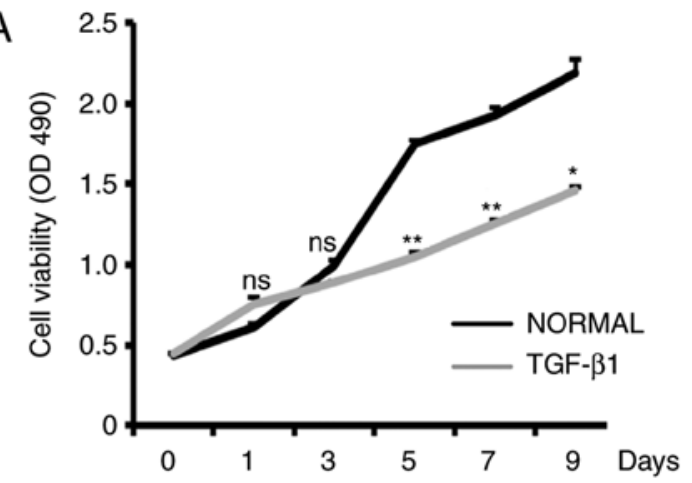

B

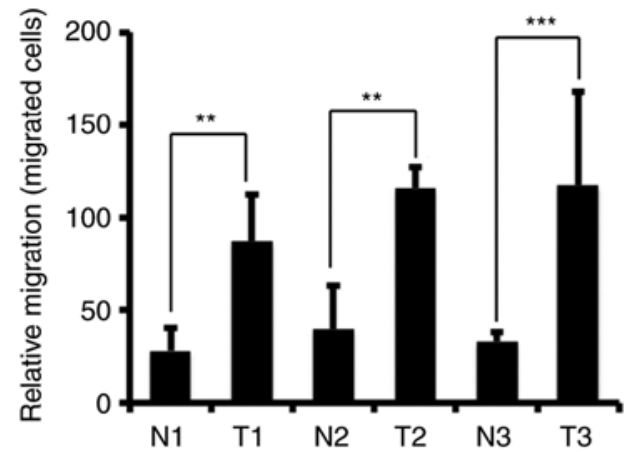

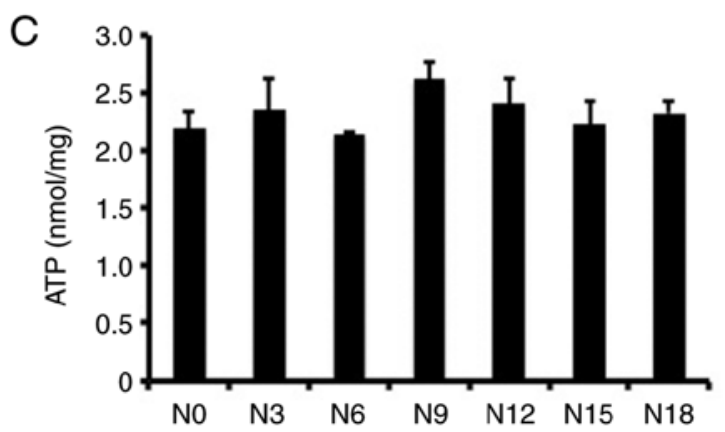

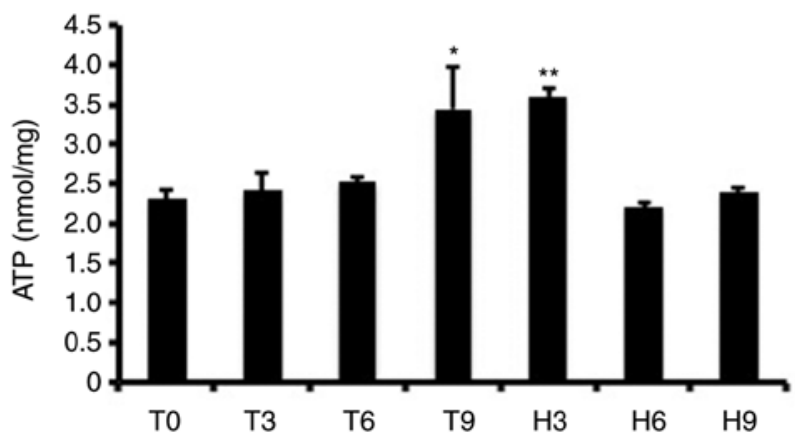

Figure 2. Decreased cell proliferation, increased cell invasion and ATP levels during TGF- $\beta 1$-induced EMT. (A) Cell viability was measured in MCF-7 cells by MTS assay after the cells were treated with TGF- $\beta 1$. (B) Representative migration assay of $2 \mathrm{ng} / \mathrm{ml}$ TGF- $\beta 1$-treated MCF-7 cells at 1,2 and 3 days. The migration of cells was quantified and is shown in the histograms. (C) ATP levels of MCF-7 cells treated for 3, 6 and 9 days with 2 ng/ml TGF- $\beta 1$. N, normal; $\mathrm{T}$, treated; $\mathrm{H}$, recovered. The data are presented as the mean $\pm \mathrm{SE}(\mathrm{n}=3) .{ }^{*} \mathrm{P}<0.05,{ }^{* *} \mathrm{P}<0.01,{ }^{* * * *} \mathrm{P}<0.001$ compared with the control cells.

during EMT, indicating that the metabolic pathway may be changed during EMT. We explored the relationship between EMT and lipid metabolism. We examined the mRNA and protein expression of lipid metabolism-related genes in MCF-7 cells after TGF- $\beta 1$-induced EMT. The RT-qPCR results showed that the expression levels of CPT1 and CD36 mRNA increased after TGF- $\beta 1$ treatment of MCF-7 cells, while the mRNA expression levels of FASN and ACC decreased (Fig. 3A-D). Western blot analysis revealed that the protein expression levels of CPT1 and CD36 increased, and the protein expression levels of FASN decreased (Fig. 3E). These results indicated that cellular fatty acid $\beta$-oxidation increased and fatty acid synthesis decreased in MCF-7 cells following EMT.

Increased OXPHOS activity during TGF- $\beta 1$-induced EMT. We examined changes in the cellular glucose metabolism pathway and OXPHOS activity. We examined the enzymes lactate dehydrogenase (LDH) and pyruvate dehydrogenase $(\mathrm{PDH})$, which are associated with glycolysis and the tricarboxylic acid (TCA) cycle, and the expression of mitochondrial oxidative phosphorylation-related proteins NDUFB8, COXI, and TFAM by western blotting. The results showed that the protein expression of LDH and PDH increased after TGF- $\beta 1$ treatment of MCF-7 cells, and the protein expression of NDUFB8, COXI and TFAM also increased compared to that in the control. However, the protein expression decreased after removal of TGF- $\beta 1$ (Fig. 4A). This indicates that glycolysis and the TCA cycle were enhanced. Subsequently, we examined changes in mtDNA copy number in MCF-7 cells during EMT. The gene content of the mitochondrial D-loop region and the
18S RNA gene were amplified by RT-qPCR, and it was found that the ratio of the mitochondrial D-loop region gene to the 18S RNA gene was significantly increased compared with that of the control cells (Fig. 4B). Fluorescent staining of mitochondrial ROS and flow cytometry analysis revealed an increase in mitochondrial ROS levels after TGF- $\beta 1$ treatment (Fig. 4C). These results indicated that intracellular mitochondrial OXPHOS activity increased in TGF- $\beta 1$-induced EMT.

The above research shows that fatty acid oxidation, oxidative phosphorylation activity and ATP levels are increased in MCF-7 cells undergoing TGF- $\beta 1$-induced EMT. It has been reported that AMPK is an energy receptor for tumor progression; in addition, the AMPK pathway also plays an important role in lipid metabolism regulation (14). Our study found that p-AMPK protein did not change before 6 days of TGF- $\beta 1$-treatment, but p-AMPK levels in treated cells were significantly higher than those in control cells on the 9th day. Moreover, the expression of ACC was decreased (Fig. 5A). The ratio of p-AMPK to total AMPK also showed an increase in p-AMPK expression level at 9 days (Fig. 5B). Therefore, we hypothesized that TGF- $\beta 1$ induces EMT in MCF-7 cells and that AMPK is activated. In addition, p-AMPK inhibited the expression of FASN and ACC, increased the expression of CPT1 and CD36, and enhanced fatty acid $\beta$-oxidation, thereby, producing a large amount of ATP to facilitate breast cancer cell metastasis (Fig. 5C).

Increased fatty acid synthesis in epithelial cells and increased fatty acid oxidation in mesenchymal cells in breast cancer tissue. Cellular experiments showed an increase in fatty acid $\beta$-oxidation and a decrease in fatty acid synthesis in the EMT 
A

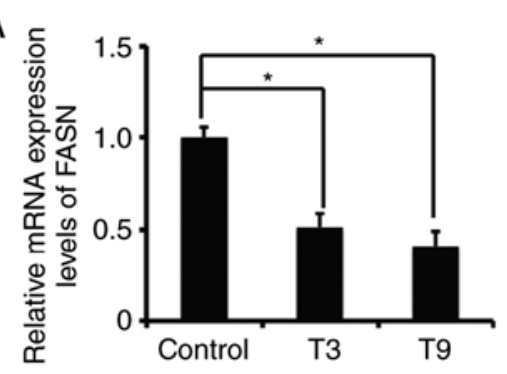

B

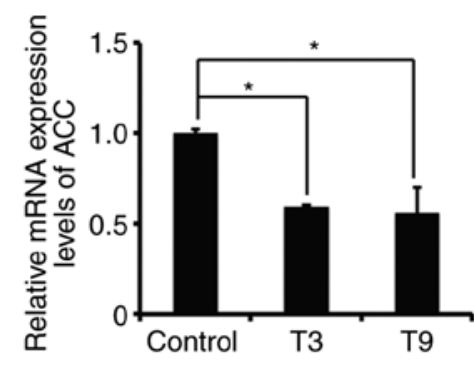

C

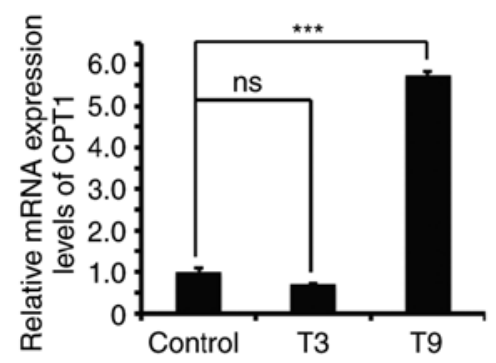

D

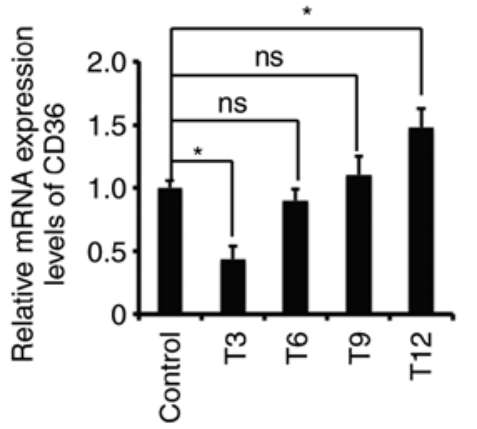

$\mathrm{E}$

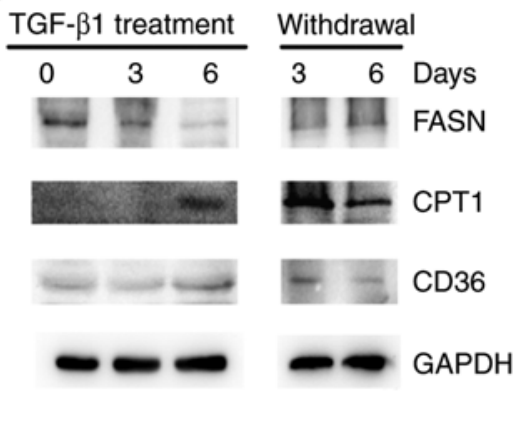

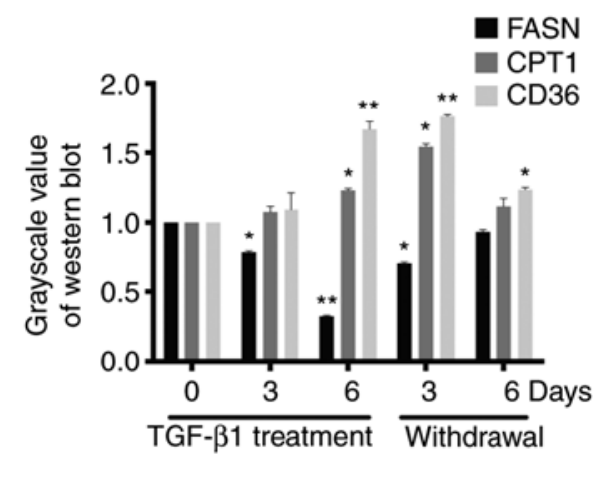

Figure 3. Increased fatty acid oxidation and decreased fatty acid synthesis in TGF- $\beta 1$-induced EMT MCF-7 cells. (A-D) mRNA expression of FASN, ACC, CPT1 and CD36 in MCF-7 cells treated at different times with $2 \mathrm{ng} / \mathrm{ml}$ TGF- $\beta 1$. mRNA levels were normalized to the $\beta$-actin levels. (E) Protein expression of FASN, CPT1 and CD36 in MCF-7 cells treated with $2 \mathrm{ng} / \mathrm{ml}$ TGF- $\beta 1$ for 0,3 and 6 days and withdrawal TGF- $\beta 1$ for 3 and 6 days were analyzed by western blotting. The protein expression was quantified and is shown in histograms. Protein levels were normalized to the GAPDH levels. The data are presented as the mean $\pm \mathrm{SE}(\mathrm{n}=3) .{ }^{*} \mathrm{P}<0.05,{ }^{* * *} \mathrm{P}<0.01,{ }^{* * * *} \mathrm{P}<0.001$ compared with the control cells.
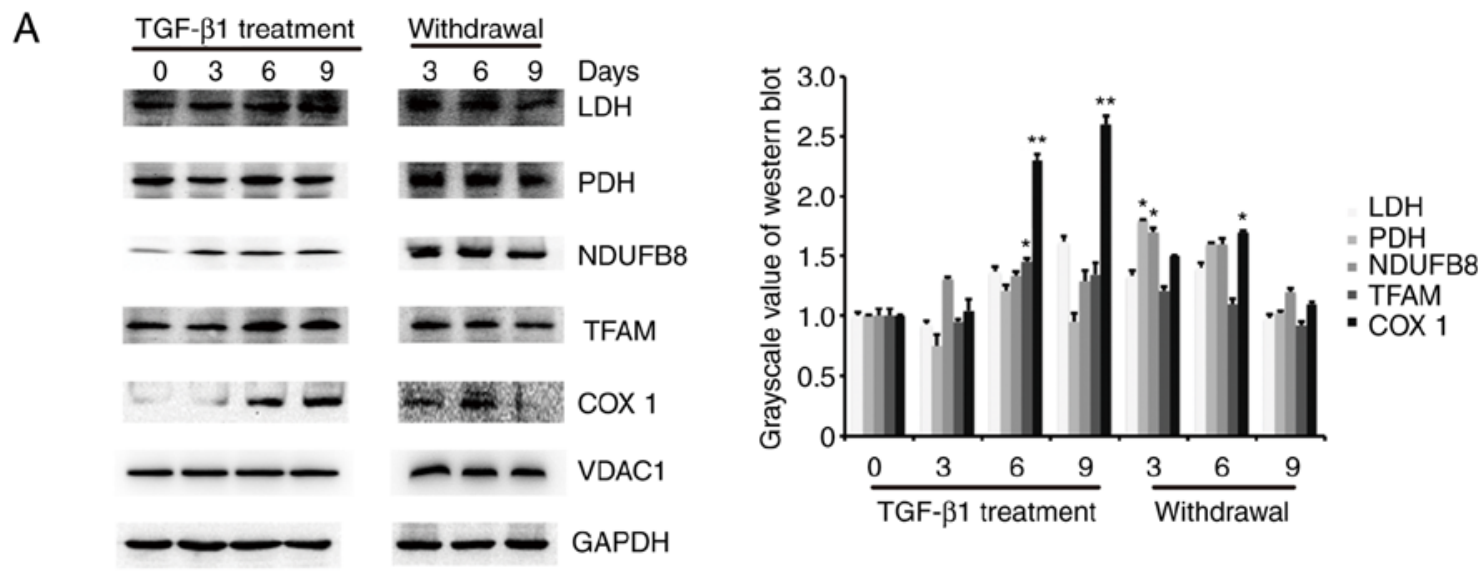

C
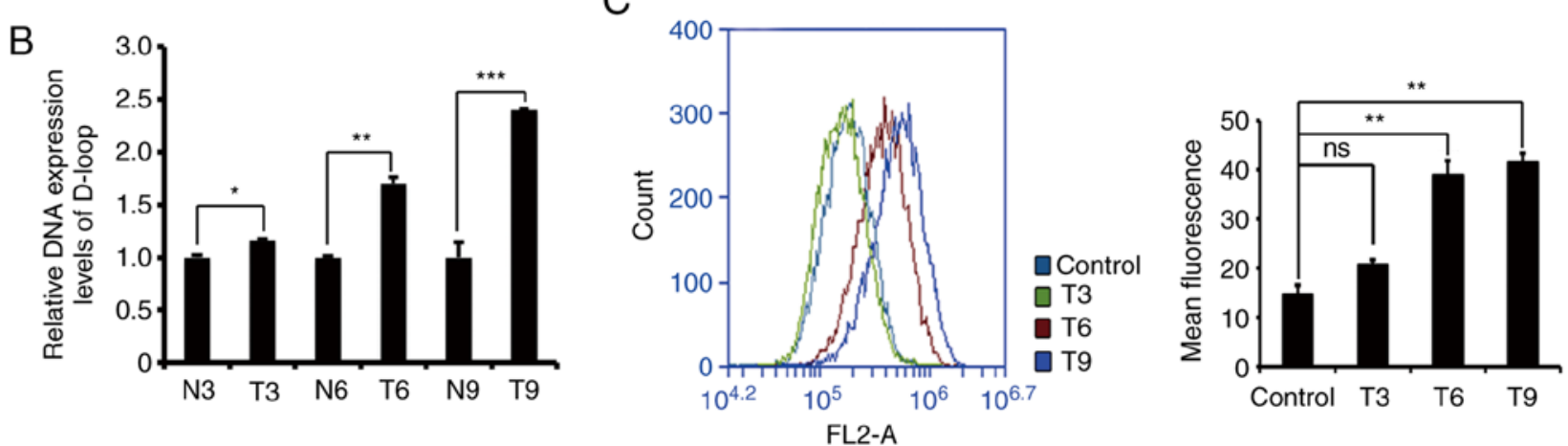

Figure 4. Increased OXPHOS activity during TGF- $\beta 1$-induced EMT. (A) Western blotting was used to detect the protein expression of LDH and PDH and of the OXPHOS-related proteins NDUFB8, COXI and TFAM in MCF-7 cells treated with $2 \mathrm{ng} / \mathrm{ml}$ TGF- $\beta 1$ for 3,6 and 9 days and withdrawal TGF- $\beta 1$ for 3, 6 and 9 days. The protein expression was quantified and is shown in histograms. (B) RT-qPCR detection of D-loop replication levels in MCF-7 cells treated with 2 ng/ml TGF- $\beta 1$ for 3, 6 and 9 days. (C) Flow cytometry was used to detect cellular ROS levels in MCF-7 cells treated with TGF- $\beta 1$ for 3, 6 and 9 days. Data are presented as the mean $\pm \mathrm{SE}(\mathrm{n}=3) .{ }^{*} \mathrm{P}<0.05,{ }^{* *} \mathrm{P}<0.01,{ }^{* * *} \mathrm{P}<0.001$ compared with the control cells. 
A
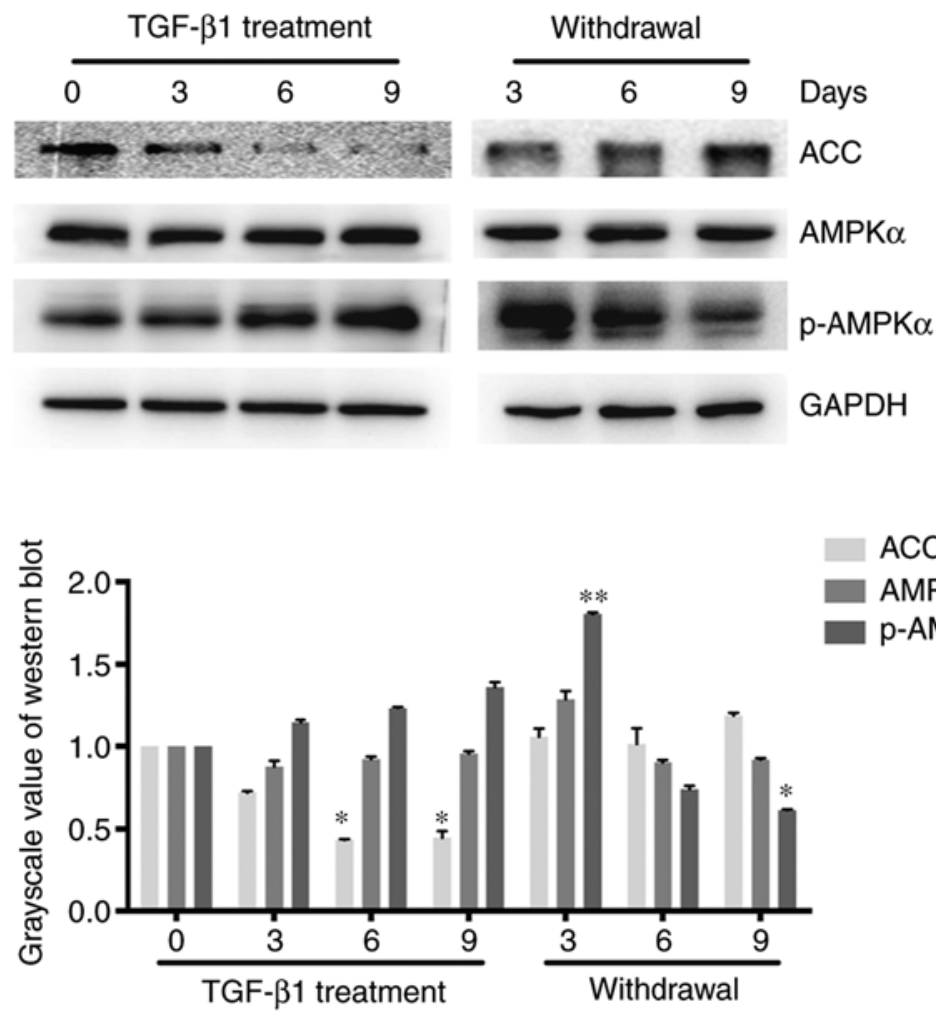

ACC

AMPK

p-AMPK

C
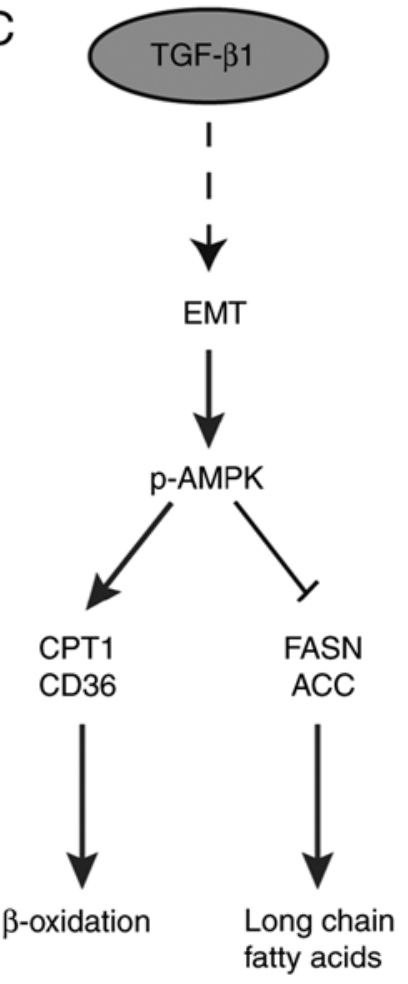

fatty acids

B

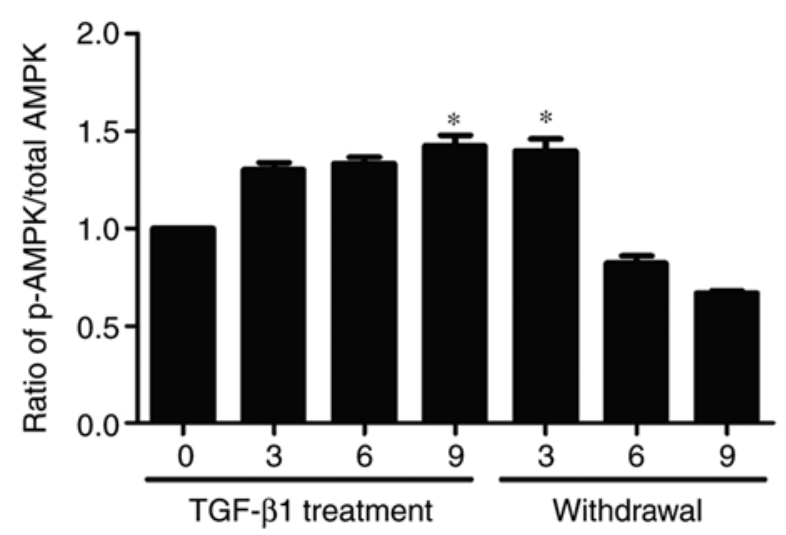

Figure 5. p-AMPK regulates fatty acid $\beta$-oxidation in TGF- $\beta 1$ induces EMT. (A) Western blot analysis of the protein expression levels of ACC, p-AMPK and AMPK. (B) The ratio of p-AMPK/total AMPK is shown in histograms. (C) The mechanism of p-AMPK regulates fatty acid metabolism in TGF- $\beta 1$ induces EMT. The data are presented as the mean $\pm \mathrm{SE}(\mathrm{n}=3) .{ }^{*} \mathrm{P}<0.05,{ }^{* * *} \mathrm{P}<0.01$ compared with the control cells.

of MCF-7 cells; thus, we examined metabolic pathway changes in breast cancer tissue. We used the epithelial cell marker E-cadherin and the mesenchymal cell marker vimentin for immunohistochemical staining in breast cancer pathological samples. The results showed that E-cadherin was expressed in epithelial cells, and vimentin was expressed in mesenchymal cells. Then, we examined the expression of FASN and CPT1 in epithelial and mesenchymal cells. The results showed that FASN was highly expressed in epithelial cells, and there were high CPT1 expression levels in interstitial cells. At the same time, the Pearson's correlation analysis shows that E-cadherin is significantly positively correlated with FASN expression $(\mathrm{P}<0.05, \mathrm{R}>0)$ and vimentin is positively correlated with CPT1 expression $(\mathrm{P}<0.05, \mathrm{R}>0$; Fig. 6A-C). These results indicated that epithelial and mesenchymal cells have different lipid metabolic pathways in breast cancer tissues. Epithelial cells mainly undergo fat synthesis, while mesenchymal cells mainly undergo lipolysis in breast cancer tissues, suggesting that mesenchymal cells need to obtain more energy through lipolysis to support cell breast cancer metastasis in breast cancer tissues.

\section{Discussion}

Reprogrammed metabolism has been identified as a hallmark of cancer cells (15). Most tumor cells are in a state of hypoxia and nutrient deficiency, and tumor cells must adapt their metabolism to survive and proliferate in harsh microenvironments (16). Cancer cells may use glucose, ketone bodies, glutamine and lactic acid as fuel sources. Lipids are also used as an important energy source in some tumor tissues (17). Chen et al, reported that the lipid metabolism 


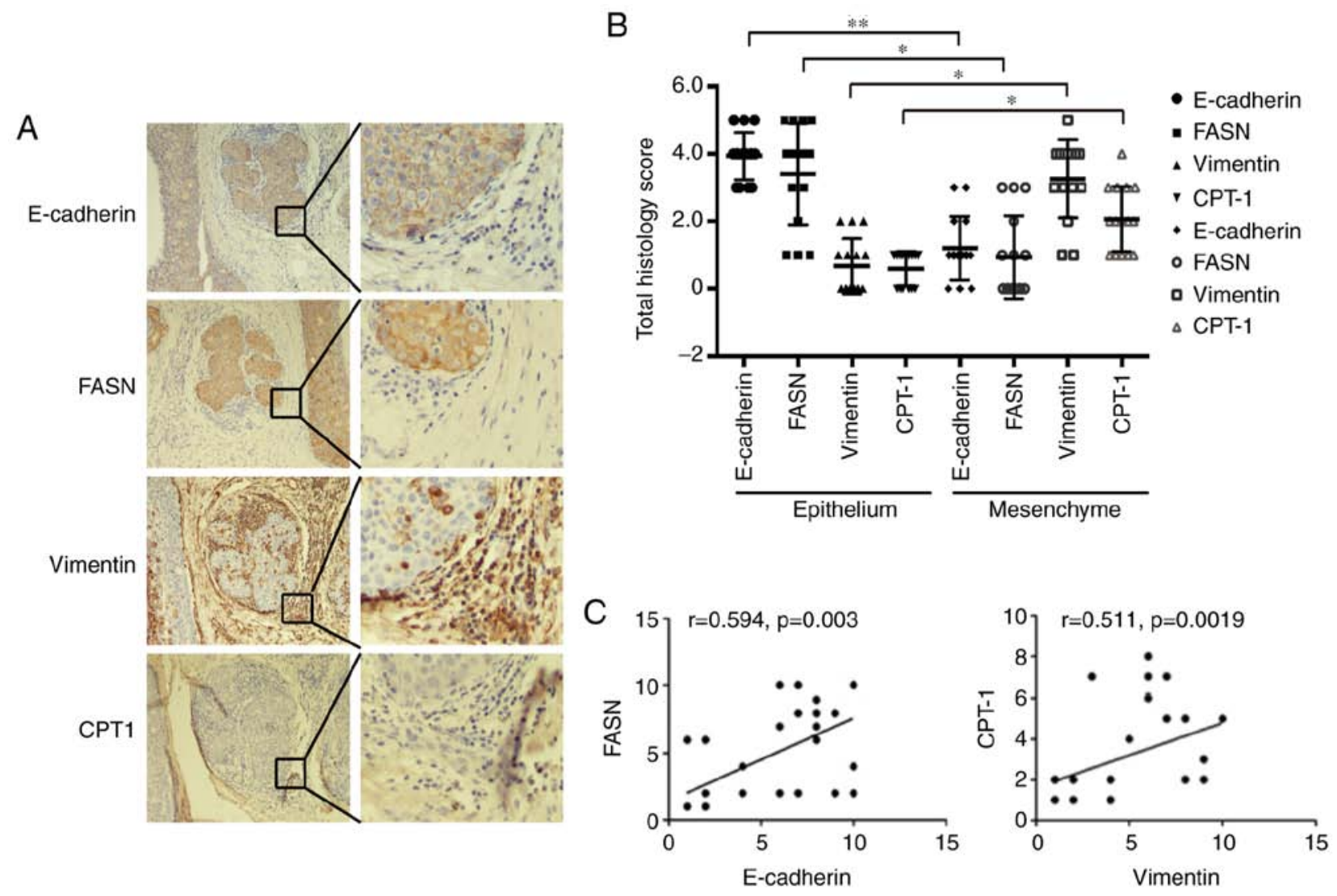

Figure 6. Increased fatty acid synthesis in epithelial cells and increased fatty acid oxidation in mesenchymal cells of breast cancer tissues. (A) Representative images of IHC staining of E-cadherin, FASN, vimentin and CPT1 protein in tumor tissues from breast cancer patients. (B) Protein expression was quantified and is shown in a scatter plot. (C) The protein expression of E-cadherin was positively correlated with the protein expression of FASN, and the protein expression of vimentin was positively correlated with the protein expression of CPT1. The data are presented as the mean $\pm \mathrm{SE}(\mathrm{n}=3)$. ${ }^{*} \mathrm{P}<0.05$, ${ }^{* *} \mathrm{P}<0.01$ compared with the control cells.

activity of ovarian cancer cells is enhanced in ascites microenvironment culture (18). Lipids affect the ability of cancer stem cells (CSCs) to self-renew (19). In our study, TGF- $\beta 1$ induced EMT in MCF-7 cells, and we detected an increase in CPT1 and CD36 expression at 9 and 12 days after TGF- $\beta 1$ treatment. A significant increase in cellular ATP levels was also detected at 9 days after TGF- $\beta 1$ treatment. The results showed that the process of TGF- $\beta 1$-induced EMT promoted the increase of fatty acid oxidation pathway to provide energy for breast cancer metastasis. Therefore, lipid metabolism plays an important role in the occurrence and metastasis of cancer.

Cancer metastasis is a prominent feature of cancer cells and is responsible for most cancer-associated mortality. Epithelial-mesenchymal transition (EMT) plays an essential role in the initiation and development of cancer metastasis. Studies showed that the acidic microenvironment derived from cancer cell metabolism induces the EMT phenotype and cancer metastasis $(20,21)$. Glycolytic enzymes (PKM2, LDHA) promoted the mesenchymal transition of cancer cells by regulating the expression of transcriptional factors $(22,23)$. In addition, EMT conversely regulates cancer metabolism $(24,25)$. However, few studies are available on fatty metabolism during EMT. In the present study, we detected changes in the expression of fatty metabolism-related enzymes during TGF- $\beta 1$-induced EMT. The results found that the expression of FASN and ACC decreased during EMT. While the expression of CPT1 and CD36 decreased after 3 days of TGF- $\beta 1$ treatment, the expression levels were significantly higher in CPT1 in T9 and in CD36 in T12 than in the control. At the same time, ATP levels also increased significantly at 9 days of TGF- $\beta 1$ treatment. We demonstrated that in the first 3 days of TGF- $\beta 1$ induced EMT, both the fatty acid anabolism and catabolism pathways were reduced, while in the later stage of EMT, the cellular catabolism of fatty acids was significantly enhanced.

AMP-activated protein kinase (AMPK) is a conserved sensor of cellular energy change that is expressed ubiquitously in eukaryotic cells (26). AMPK responds to changes in intracellular adenine nucleotide levels and is activated by an increase in the ratio of AMP/ADP relative to ATP (27). Furthermore, AMPK is very important in breast cancer glycolysis and glycogen catabolism. Findings have shown that, the glycolysis inhibitor 2-deoxyglucose (2-DG) activates the expression of AMPK by decreasing the ATP content, thus contributing to the recycling of ATP in MCF-7 cells (28). In addition, it has been reported that p-AMPK regulates the fatty acid metabolism pathway. When the ATP level is low, AMPK is activated, and p-AMPK inhibits ACC expression (29,30). In the present study, we detected that p-AMPK increased significantly at 9 days of TGF- $\beta 1$ treatment. Thus, changes in energy levels occur during TGF- $\beta 1$-induced EMT in MCF-7 cells. At the later stages of epithelial to mesenchymal transition, ATP was 
consumed, ATP content decreased, p-AMPK was activated, and fatty acid oxidation and oxidative phosphorylation were promoted. ATP was produced, and cells provided energy for the distant metastasis of cancer. However, whether the energy level during breast cancer EMT is a signal to regulate lipid metabolism needs further verification. Further studies should investigate the effect of ATP level in the regulation of fatty acid oxidation during EMT in breast cancer including the AMPK inhibitor experiment.

In conclusion, to the best of our knowledge, this study has shown, for the first time, that fatty acid $\beta$-oxidation and oxidative phosphorylation may be regulated by the p-AMPK pathway during EMT. Fatty acids act as an energy source to promote breast cancer cell metastasis. The results of the present study are important for understanding the relationship between cancer metabolism and metastasis during EMT in breast cancer cells.

\section{Acknowledgements}

Not applicable.

\section{Funding}

This study was financially supported by the National Nature Science Foundation of China (grant nos. 31760331, 31260276, $31601155,81860531,81660583,81560458$ and 81760507), the Science and Technology Innovation Team of Yunnan Province (2011CI123) and Dali University (ZKPY2019308), the Reserve Talents of Young and Middle-aged Academic and Technical Leaders of Yunnan Province (grant no. 2017HB077), the Top Young Talents of Ten Thousand Talents Plan of Yunnan Province (grant no. 2019), and the Key Project of Science and Technology Department of Yunnan Province (grant no. 2020).

\section{Availability of data and materials}

The datasets used or analyzed in the present study are available from the corresponding author upon reasonable request.

\section{Authors' contributions}

QQL, MY and WX conceived and designed the experiments. QQL, HYH, SA, XXY, TL, LY and ZYF performed the experiments. ZQZ, LD, QHC and JL were responsible for the collection and assembly of data. QQL and SA analyzed the data and wrote the manuscript. All authors read and approved the final manuscript.

\section{Ethics approval and consent to participate}

All procedures were conducted in accordance with standard guidelines for the Study of Ethics Committee of Yunnan University (Kunming, China), and written informed consent was obtained. Prior to study inclusion, written informed consent was obtained from all the patients.

\section{Patient consent for publication}

Not applicable.

\section{Competing interests}

The authors declare that they have no competing interests.

\section{References}

1. Anastasiadi Z, Lianos GD, Ignatiadou E, Harissis HV and Mitsis M: Breast cancer in young women: An overview. Updates Surg 69: 313-317, 2017.

2. Medeiros B and Allan AL: Molecular mechanisms of breast cancer metastasis to the lung: Clinical and experimental perspectives. Int J Mol Sc 20: 2272, 2019.

3. Minn AJ, Gupta GP, Siegel PM, Bos PD, Shu W, Giri DD, Viale A, Olshen AB, Gerald WL and Massagué J: Genes that mediate breast cancer metastasis to lung. Nature 436: 518-524, 2005.

4. Hu J, Li G, Zhang P, Zhuang X and Hu G: A CD44v ${ }^{+}$subpopulation of breast cancer stem-like cells with enhanced lung metastasis capacity. Cell Death Dis 8: e2679, 2017.

5. Xu X, Yan Q, Wang Y and Dong X: NTN4 is associated with breast cancer metastasis via regulation of EMT-related biomarkers. Oncol Rep 37: 449-457, 2017.

6. Pastushenko I, Brisebarre A, Sifrim A, Fioramonti M, Revenco T, Boumahdi S, Van Keymeulen A, Brown D, Moers V, Lemaire $\mathrm{S}$, et al: Identification of the tumour transition states occurring during EMT. Nature 556: 463-468, 2018.

7. Nieto MA, Huang RY, Jackson RA and Thiery JP: EMT: 2016. Cell 166: 21-45, 2016.

8. Ungefroren $\mathrm{H}$, Witte D and Lehnert H: The role of small GTPases of the Rho/Rac family in TGF- $\beta$-induced EMT and cell motility in cancer. Dev Dyn 247: 451-461, 2018.

9. Liu FL, Mo EP, Yang L, Du J, Wang HS, Zhang H, Kurihara H, $\mathrm{Xu} \mathrm{J}$ and Cai SH: Autophagy is involved in TGF- $\beta 1$-induced protective mechanisms and formation of cancer-associated fibroblasts phenotype in tumor microenvironment. Oncotarget 7 : 4122-4141, 2016

10. Huang R and Zong X: Aberrant cancer metabolism in epithelialmesenchymal transition and cancer metastasis: Mechanisms in cancer progression. Crit Rev Oncol Hematol 115: 13-22, 2017.

11. Chen XL, Lei L, Hong LL and Ling ZQ: Potential role of NDRG2 in reprogramming cancer metabolism and epithelialto-mesenchymal transition. Histol Histopathol 33: 655-663, 2018.

12. Tan TZ, Miow QH, Miki Y, Noda T, Mori S, Huang RY and Thiery JP: Epithelial-mesenchymal transition spectrum quantification and its efficacy in deciphering survival and drug responses of cancer patients. EMBO Mol Med 6: 1279-1293, 2014.

13. Syed V: TGF- $\beta$ Signaling in Cancer. J Cell Biochem 117: 1279-1287, 2016

14. Kim I and He YY: Targeting the AMP-activated protein kinase for cancer prevention and therapy. Front Oncol 3: 175, 2013.

15. Chiarugi P and Cirri P: Metabolic exchanges within tumor microenvironment. Cancer Lett 380: 272-280, 2016.

16. Gouirand V, Guillaumond F and Vasseur S: Influence of the tumor microenvironment on cancer cells metabolic reprogramming. Front Oncol 8: 117, 2018.

17. Cheng S, Wang G, Wang Y, Cai L, Qian K, Ju L, Liu X, Xiao $Y$ and Wang X: Fatty acid oxidation inhibitor etomoxir suppresses tumor progression and induces cell cycle arrest via PPAR $\gamma$-mediated pathway in bladder cancer. Clin Sci (Lond) 133: 1745-1758, 2019.

18. Chen RR, Yung MMH, Xuan Y, Zhan S, Leung LL, Liang RR, Leung THY, Yang H, Xu D, Sharma R, et al: Targeting of lipid metabolism with a metabolic inhibitor cocktail eradicates peritoneal metastases in ovarian cancer cells. Commun Biol 2: 281, 2019.

19. Li J, Condello S, Thomes-Pepin J, Ma X, Xia Y, Hurley TD, Matei D and Cheng JX: Lipid desaturation is a metabolic marker and therapeutic target of ovarian cancer stem cells. Cell Stem Cell 20: 303-314.e5, 2017.

20. Klein M, Seeger P, Schuricht B, Alper SL and Schwab A: Polarization of $\mathrm{Na}(+) / \mathrm{H}(+)$ and $\mathrm{Cl}(-) / \mathrm{HCO}(3)(-)$ exchangers in migrating renal epithelial cells. J Gen Physiol 115: 599-608, 2000.

21. Lagana A, Vadnais J, Le PU, Nguyen TN, Laprade R, Nabi IR and Noël J: Regulation of the formation of tumor cell pseudopodia by the $\mathrm{Na}(+) / \mathrm{H}(+)$ exchanger NHE1. J Cell Sc 113: 3649-3662, 2000. 
22. Fan FT, Shen CS, Tao L, Tian C, Liu ZG, Zhu ZJ, Liu YP, Pei CS Wu HY, Zhang L, et al: PKM2 regulates hepatocellular carcinoma cell epithelial-mesenchymal transition and migration upon EGFR activation. Asian Pac J Cancer Prev 15: 1961-1970, 2014.

23. Arseneault R, Chien A, Newington JT, Rappon T, Harris R and Cumming RC: Attenuation of LDHA expression in cancer cells leads to redox-dependent alterations in cytoskeletal structure and cell migration. Cancer Lett 338: 255-266, 2013.

24. Liu X, Wang X, Zhang J, Lam EK, Shin VY, Cheng AS, Yu J, Chan FK, Sung JJ and Jin HC: Warburg effect revisited: An epigenetic link between glycolysis and gastric carcinogenesis. Oncogene 29: 442-450, 2010.

25. Yang L, Hou Y, Yuan J, Tang S, Zhang H, Zhu Q, Du YE Zhou M, Wen S, Xu L, et al: Twist promotes reprogramming of glucose metabolism in breast cancer cells through PI3K/AKT and p53 signaling pathways. Oncotarget 6: 25755-25769, 2015.
26. Hardie DG, Schaffer BE and Brunet A: AMPK: An energysensing pathway with multiple inputs and outputs. Trends Cell Biol 26: 190-201, 2016.

27. Carling D: AMPK signalling in health and disease. Current opinion in cell biology 45: 31-37, 2017

28. Wu Y, Sarkissyan M, McGhee E, Lee S and Vadgama JV: Combined inhibition of glycolysis and AMPK induces synergistic breast cancer cell killing. Curr Opin Cell Biol 151: 529-539, 2015.

29. Liu X, Chhipa RR, Nakano I and Dasgupta B: The AMPK inhibitor compound $\mathrm{C}$ is a potent AMPK-independent antiglioma agent. Mol Cancer Ther 13: 596-605, 2014.

30. Mauro L, Naimo GD, Gelsomino L, Malivindi R, Bruno L, Pellegrino M, Tarallo R, Memoli D, Weisz A, Panno ML and Andò S: Uncoupling effects of estrogen receptor alpha on LKB1/AMPK interaction upon adiponectin exposure in breast cancer. FASEB J 32: 4343-4355, 2018. 